\title{
Síndrome de Leriche en un paciente con síndrome antifosfolípido catastrófico
}

\section{Leriche Syndrome in a Patient with Catastrophic Antiphospholipid Syndrome}

\author{
Mauricio Rangel Hernández ${ }^{1}$
}

${ }^{1}$ Escuela de Posgrados en Sanidad Naval, Universidad Naval,
Secretaría de Marina Armada de México, Ciudad de México, México

Rev Argent Radiol 2018;82:178-180.

Estimados Editores,

El síndrome de anticuerpos antifosfolípidos (SAAF), es una rara condición, caracterizada por trombosis arterial y/o venosa, acompañada por trombocitopenia de leve a moderada y títulos altos de anticuerpos antifosfolípidos. En mujeres se expresa en una pérdida fetal recurrente, se presenta con una incidencia de 5 casos nuevos por cada 100.000 personas y una prevalencia de $1-5 \%$, en su presentación catastrófica. La misma consiste en una falla orgánica múltiple con los siguientes eventos clínicos: evidencia clínica de afección multiorgánica desarrollada en un periodo de tiempo corto y evidencia histopatológica de oclusión de vasos de pequeño calibre. ${ }^{1}$ A pesar de la baja incidencia de la presentación catastrófica, menos de $1 \%$ de los pacientes con SAAF, la mayoría de casos donde se desarrolla tienen desenlaces fatales.2 Aún se desconoce por qué algunos pacientes presentan oclusión vascular en grandes vasos mientras otros desarrollan trombosis de repetición en vasos de pequeño calibre. Debido a la rareza de ese síndrome, el Foro Europeo de Anticuerpos Antifosfolipídicos creó en el año 2000, una base de datos en la cual actualmente existe un registro de alrededor de 500 pacientes. $^{3}$

Por otro lado, la enfermedad oclusiva aortoilíaca (EOA) o síndrome de Leriche se define típicamente como la oclusión de la aorta abdominal por debajo de las arterias renales, aunque en distintos reportes incluyen la oclusión frecuente de la arteria renal derecha, ${ }^{4}$ secundaria a la obstrucción de la arteria aorta abdominal y las arterias ilíacas comunes, secundaria a trombosis, por coágulos organizados y placas ateromatosas. Clínicamente se caracteriza por la presencia de disfunción eréctil, claudicación intermitente, atrofia de los músculos de los miembros inferiores, disminución de los pulsos femorales ${ }^{5}$ y palidez de piernas y pies, ${ }^{6}$ predominantemente en adultos

received

October 9, 2017

accepted

December 10, 2017

published online

May 16, 2018
Address for correspondence Mauricio Rangel Hernández, Residente de 3er año de Radiología e Imagen, Escuela de Posgrados en Sanidad Naval, Universidad Naval, Secretaría de Marina Armada de México, Ciudad de México, México (e-mail: mrh850610@gmail.com).

jóvenes masculinos, aunque se han reportado varios casos en mujeres jóvenes. Por otra parte, el espectro trombótico oclusivo ha sido reportado en múltiples arterias como la mesentérica superior e inferior. ${ }^{7}$ A pesar de la apariencia devastadora de ese síndrome, la circulación sanguínea no se interrumpe por completo, pues esos pacientes generan vías colaterales de flujo sanguíneo que irrigan las extremidades distales. No obstante, generalmente las vías colaterales formadas por anastomosis término de arterias grandes, ofrecen menor flujo sanguíneo. ${ }^{8}$ La circulación colateral consiste en vasos aferentes originados en proximidad a la oclusión, que se anastomosan con vasos eferentes distales a la oclusión. ${ }^{9}$ Los vasos ocluidos reciben flujo anterógrado proximal a la oclusión y flujo retrógrado distalmente; sin embargo hay una tendencia a la angiogénesis de vías colaterales de pequeño calibre para minimizar la resistencia al flujo, ${ }^{10}$ por ejemplo en la vía colateral de Winslow, que permite la irrigación desde la arteria subclavia a través de la arteria torácica interna, epigástrica superior e inferior y finalmente a las arterias ilíaca externa y femoral común.

Se realizó una búsqueda avanzada en PubMed empleando los términos Leriche syndrome y antiphospholipid syndrome, obteniendo tres resultados, de los cuales solamente dos son concordantes con pacientes con EOA y SAAF como reportes de caso. No encontramos literatura, ni investigaciones médicas que relacionen la fisiopatología a esas entidades clínicas; sin embargo, existen similitudes patológicas que abren una duda razonable para su investigación, por ejemplo, el hecho de compartir un origen autoinmune, con estados protrombóticos.

En nuestro caso, encontramos un paciente masculino de 56 años al momento de su fallecimiento, sin antecedentes de enfermedades crónico degenerativas, ni reumatológicas familiares, tabaquista a razón de un índice tabáquico de 33
Copyright (c) 2019, Sociedad Argentina de Radiología. Publicado por Thieme Revinter Publicações Ltda., Rio de Janeiro, Brazil. Todos los derechos reservados.

\section{License terms}

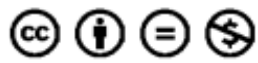

$10.1055 / \mathrm{s}-0038-1639497$ ISSN 1852-9992. 
(1 paquete diario desde los 15 años). Los síntomas comenzaron a manifestarse a los 48 años, mostrando un cuadro clínico con claudicación y edema del miembro inferior izquierdo, que requirió hospitalización, con un diagnóstico de trombosis venosa profunda de venas tibiales y poplítea, realizando el tratamiento y posterior alta. En los siguientes seis meses presentó dos eventos de trombosis venosa en miembros inferiores y uno en miembro superior derecho, diagnosticados por ultrasonido. Para completar el protocolo de estudio, se realizaron análisis de laboratorio, mostrando como resultado: AT-III 29.5mg/dl, Anticuerpo (AC) ANTICARDIOLIPINA IgG101 GPL U/Ml, AC ANTICARDIOLIPINA IgA16 APL U/Ml, AC ANTICA RDIOLIPINA IgM1 MPL U/Ml; que llevó a su diagnóstico de SAAF. Se derivó a los servicios de hematología, cirugía vascular y reumatología, que indicaron tratamiento médico, sin embargo, el paciente suspendió el tratamiento farmacológico establecido y hubo inasistencia a consultas de control.

Siete años después del diagnóstico de SAAF, el paciente reingresó a la unidad de cuidados críticos por presentar encefalopatía hepática, ascitis, oliguria e incremento en las troponinas, así como úlceras vasculares, con cambio de coloración en tercio distal de ambos miembros inferiores, y falla orgánica múltiple, por lo que se decidió realizar una tomografía computada multidetector con contraste, donde se observó oclusión total de la aorta abdominal (-Fig. 1). También se detectó oclusión de las arterias ilíacas comunes e internas y la arteria renal izquierda (- Fig. 2). Se observó la circulación colateral que irrigaba el miembro inferior izquierdo a través de la arteria ilíaca externa que daba flujo a la arteria femoral ipsilateral (-Fig. 3). También se pudo apreciar la irrigación colateral de la porción distal de la

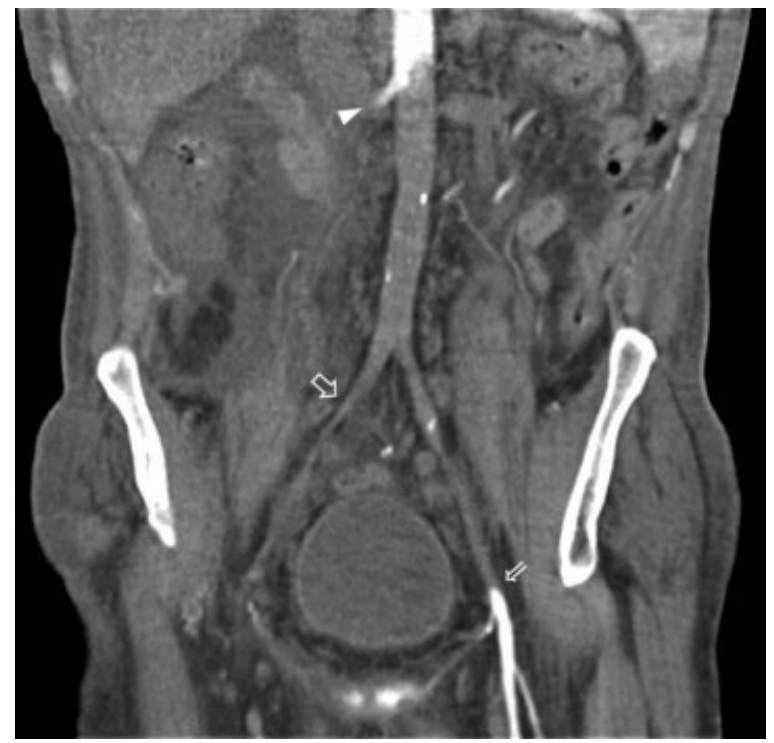

Fig. 1 Oclusión total de la arteria aorta abdominal. Tomografía abdominal en fase arterial, con reconstrucción multiplanar (MPR), curva coronal en promedio de intensidad de proyección (average intensity project, AIP) mostrando obstrucción de la arteria aorta y la arteria renal izquierda con permeabilidad de la arteria renal derecha (cabeza de flecha); nótese la obstrucción total de la arteria ilíaca externa derecha (flecha vacía) y permeabilidad de la porción distal de las arterias ilíaca externa y femoral izquierda (flecha angosta).

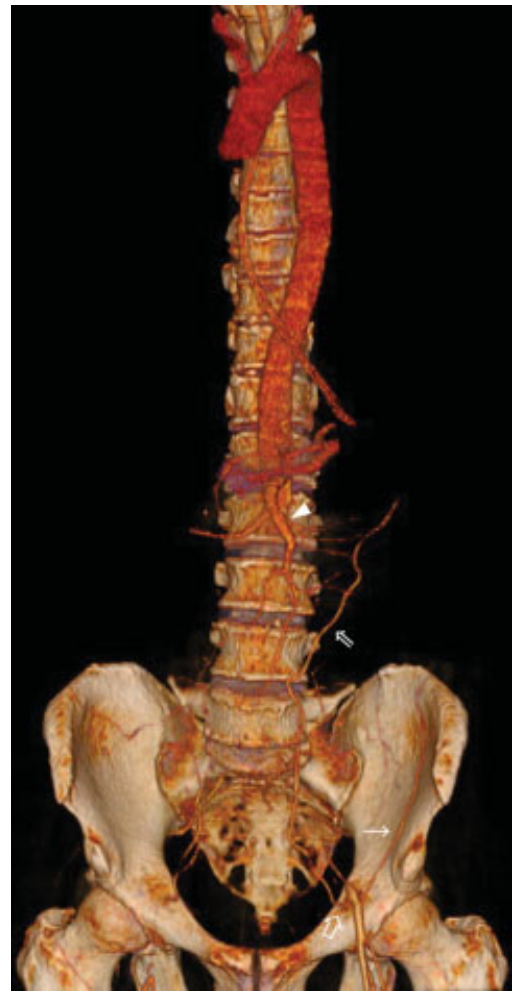

Fig. 2 Vía colateral de Winslow. Tomografía con reconstrucción volumétrica mostrando obstrucción total de la aorta abdominal, por debajo de la arteria mesentérica superior (cabeza de flecha), y el sitio de anastomosis (flecha vacía) de la arteria epigástrica inferior (flecha abierta), con la porción distal de la arteria ilíaca externa, que forma una vía colateral con la arteria torácica interna, vía la arteria epigástrica superior (no mostradas), y la arteria circunfleja profunda (flecha angosta).

arteria ilíaca externa por la arteria circunfleja profunda, conformando la vía distal de Winslow.

A pesar de que la mejor evaluación se realiza con angiografía por sustracción digital, la tomografía contrastada es un método más accesible. Sin embargo, en pacientes en los que no se

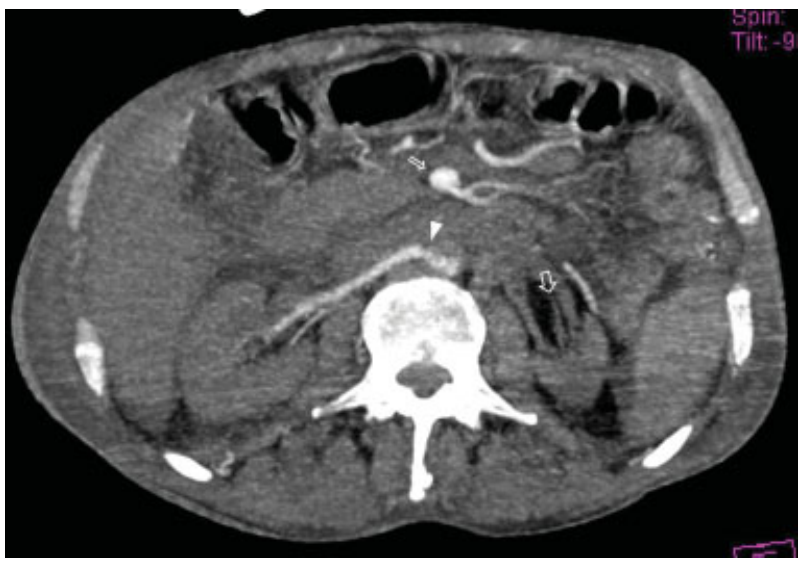

Fig. 3 Riñón izquierdo hipotrófico. Tomografía en fase arterial, que muestra oclusión parcial de la aorta a nivel de la bifurcación de la arteria renal derecha (cabeza de flecha). Ella presenta flujo conservado; hipotrofia renal izquierda con oclusión de la arteria (flecha vacía). Nótese la arteria mesentérica prominente (flecha abierta), compensando el flujo arterial abdominal. 
puede realizar una tomografía con contraste, una opción viable es la angioresonancia contrastada. En cualquiera de los casos, el diagnóstico de SAAF será clínico, y el de la EOA se realizará por la presencia de la oclusión aortoilíaca usualmente por debajo de las arterias renales.

\section{Confidencialidad de los datos}

Los autores declaran que han seguido los protocolos de su centro de trabajo sobre la publicación de datos de pacientes y que todos los pacientes incluidos en el estudio han recibido información suficiente $\mathrm{y}$ han dado su consentimiento informado por escrito para participar en dicho estudio.

Conflicto de Intereses

Los autores del trabajo declaran no tener ningún conflicto de intereses.

\section{Bibliografía}

1 Cervera R. Antiphospholipid syndrome. Thromb Res 2017;151 (Suppl 1):S43-S47
2 Rodriguez-Pintó I, Espinosa G, Cervera R. Catastrophic antiphospholipid syndrome: The current management approach. Best Pract Res Clin Rheumatol 2016;30(02):239-249

3 Santos TDS, Ieque AL, de Carvalho HC, et al. Antiphospholipid syndrome and recurrent miscarriage: A systematic review and meta-analysis. J Reprod Immunol 2017;123:78-87

4 Takigawa M, Akutsu K, Kasai S, et al. Angiographic documentation of aortoiliac occlusion in Leriche's syndrome. Can J Cardiol 2008; 24(07):568

5 Jawor WJ, Plice SG. Thrombotic obliteration of the abdominal aorta; report of a case. J Am Med Assoc 1952;149(02):142-143

6 Leriche R, Morel A. The syndrome of thrombotic obliteration of the aortic bifurcation. Ann Surg 1948;127(02):193-206

7 Ruehm SG, Weishaupt D, Debatin JF. Contrast-enhanced MR angiography in patients with aortic occlusion (Leriche syndrome). J Magn Reson Imaging 2000;11(04):401-410

8 Ahmed S, Raman SP, Fishman EK. CT angiography and 3D imaging in aortoiliac occlusive disease: collateral pathways in Leriche syndrome. Abdom Radiol (NY) 2017;42(09):2346-2357

9 Oliveira N, Oliveira F, Dias E, Borges L, Cássio I. Leriche syndrome in a patient with situs inversus totalis. Ann Vasc Surg 2015;29 (06):1317.e5-1317.e8

10 Sebastià C, Quiroga S, Boyé R, Perez-Lafuente M, Castellà E, AlvarezCastells A. Aortic stenosis: spectrum of diseases depicted at multisection CT. Radiographics 2003;23(Spec No):S79-S91 